\title{
Le choix du métier
}

Typologie des trajectoires menant vers l'enseignement dans le canton de Vaud en Suisse

The choice of the profession. Typology of the pathways leading to teaching in the canton of Vaud in Switzerland

La elección del oficio. Tipología de las trayectorias que conducen hacia la enseñanza en el cantón de Vaud en Suiza

\section{Crispin Girinshuti}

\section{OpenEdition}

\section{Journals}

Édition électronique

URL : https://journals.openedition.org/ries/5806

DOI : 10.4000/ries.5806

ISSN : 2261-4265

Éditeur

France Education international

Édition imprimée

Date de publication : 1 avril 2017

Pagination : 79-88

ISBN : 978-2-85420-614-2

ISSN : 1254-4590

\section{Référence électronique}

Crispin Girinshuti, « Le choix du métier », Revue internationale d'éducation de Sèvres [En ligne], 74 | avril 2017, mis en ligne le 01 avril 2019, consulté le 11 mars 2022. URL : http://journals.openedition.org/ ries/5806 ; DOI : https://doi.org/10.4000/ries.5806 


\title{
Le choix du métier
}

\section{Typologie des trajectoires menant vers l'enseignement dans le canton de Vaud en Suisse}

\author{
Crispin Girinshuti \\ Haute école pédagogique du canton de Vaud, \\ Suisse
}

En Suisse, l'intérêt pour la question du choix du métier d'enseignant répond à une préoccupation politique observée globalement dans le monde : une pénurie qui frappe le métier, dont l'attrait diminue. Deux études quantitatives se sont saisies de cet objet pour en proposer une lecture généralisable au contexte suisse de l'enseignement obligatoire (primaire et secondaire 1) d'une part (Denzler \& Wolter, 2009), et de l'enseignement professionnel suisse (secondaire 2 orienté métier) d'autre part (D'Ascoli \& Berger, 2012). Les premiers auteurs ont mis en évidence l'importance de l'origine socioéconomique, du genre, du degré d'enseignement visé, mais également de la disposition territoriale des institutions de formation. L'analyse de D'Ascoli et Berger démontre, quant à elle, que ceux qui choisissent l'enseignement professionnel sont mus par des motivations intrinsèques, d'utilité sociale ou d'utilité personnelle, qui varient notamment selon la région linguistique, le sexe et le taux d'activité.

Notre intérêt pour la question du choix de l'enseignement s'inscrit dans une problématique plus large de compréhension de l'insertion professionnelle des enseignants des degrés primaire, secondaire 1 et secondaire 2 en Suisse romande. Notre thèse (Girinshuti, 2014) est que l'insertion professionnelle de ces individus est un processus qui se déroule en trois étapes : le choix du métier - objet du présent article -, l'accès à l'emploi et l'insertion subjective ${ }^{1}$. Nous soutenons l'idée que ce processus s'inscrit dans une "carrière ", au sens de la sociologie interactionniste (Hughes, 1996). Dans l'étude du choix de l'enseignement, les individus se distinguent notamment par leur "état de carrière ", qui est la variabilité du lien entre le cycle de la vie biologique et le cycle de la vie sociale à un moment donné de leur parcours. Ce postulat met notamment en exergue l'âge, qui est la première dimension de la notion de carrière, selon Hughes.

C'est dans une telle optique que nous avons effectué une analyse qualitative de contenu d'entretiens rétrospectifs de parcours de vie et de formation de 45 individus en dernière année de formation, à la haute école pédagogique du canton de Vaud, en 2013. En effet, celle-ci montre que le choix du métier s'est construit socialement jusqu'au moment où ces individus ont commencé

1. Le sentiment d'insertion exprimé par la personne qui occupe un métier selon des critères qui dépassent les seules considérations objectives d'un emploi stable (type de contrat, protection de l'emploi, salaire, etc.). 
leur formation à l'enseignement. En fait, leur rapport à l'enseignement est fonction de leur état de carrière au moment de commencer cette formation à l'enseignement. Cet article met en lumière ce qui singularise les trois principales trajectoires vers l'enseignement que nous avons identifiées : (1) les trajectoires linéaires ou directes, (2) les trajectoires indirectes et (3) les trajectoires dites «second métier».

Les parcours linéaires vers l'enseignement regroupent les trajectoires d'individus dont les études les ont menés directement à la formation à l'enseignement. Leur expérience sur le marché de travail relève du "job d'étudiant ", mais celle-ci participe à une socialisation anticipatrice à l'activité d'enseignement (Charles, 2012). Les valeurs qui les attachent au métier trouvent leur origine dans leur "socialisation primaire spécifique»(Charles, 2012), notamment au travers de leur propre vécu en tant qu'élève.

Avec les trajectoires indirectes, l'orientation vers l'enseignement s'effectue à la suite d'un premier projet professionnel contrarié. Le métier s'impose comme alternative providentielle, par les mêmes éléments de socialisation constatés auprès de ceux qui choisissent le métier directement.

Les personnes plus âgées des cohortes de formation à l'enseignement se sont pour la plupart orientées vers l'enseignement en tant que second métier. Cette catégorie est celle qui est la plus proche de la population analysée par D'Ascoli et Berger (2012). En premier lieu, elles se caractérisent par le lien entre le premier métier exercé et l'enseignement, et en second lieu, par la place subjective de l'enseignement dans leur quotidien.

\section{LES TRAJECTOIRES LINÉAIRES VERS L'ENSEIGNEMENT Les plus jeunes des cohortes de diplômés}

Les individus crédités d'un parcours linéaire vers l'enseignement ont une trajectoire de formation qui les mène directement vers la formation à l'enseignement. À l'instar des résultats de Denzler et Wolter (2009), une première distinction systémique s'observe entre ceux qui choisissent la formation à l'enseignement primaire, sans la nécessité de passer par l'université, et les futurs enseignants du secondaire, dont les prérequis de formation nécessitent ce passage. En effet, un diplôme de niveau secondaire 2 - une maturité fédérale ou spécialisée - donne accès à la formation à l'enseignement primaire dans les instituts de formation ${ }^{2}$. Pour une formation à l'enseignement secondaire, les candidats doivent acquérir au préalable au moins un bachelor universitaire ${ }^{3}$ dans les disciplines d'enseignement souhaitées.

2. Dans certains cantons suisse, cette formation fait partie intégrante des cursus de l'université.

3. Un certain nombre de crédit ECTS est nécessaire pour valider une discipline au niveau secondaire 1. Pour suivre une formation qui donne accès à l'enseignement post-obligatoire (secondaire 2), un master universitaire est exigé. 
Treize femmes ont une trajectoire qui les a directement menées vers l'enseignement primaire. Leur âge médian est de 23 ans. Au moment de l'entretien, la plus jeune avait 21 ans et la plus âgée 24 . Quatre femmes et trois hommes ${ }^{4}$ ont suivi une formation universitaire, avant de suivre une formation d'enseignants du secondaire. Leur âge médian est de 27 ans. La plus jeune, titulaire d'un bachelor universitaire, a 25 ans au moment de l'interview, tandis que la plus âgée, 30 ans, est licenciée en lettres. En termes de "carrière ", ces âges sont à mettre en lien avec le parcours scolaire que ces enseignants ont suivi. Ainsi, la trajectoire de la plus âgée illustre les possibilités offertes par le système scolaire vaudois. Son parcours de formation est plus long, notamment parce qu'elle a dû emprunter des passerelles afin d'accéder à l'Université 5 .

\section{L'enseignement comme " phénomène de continuation "}

Dans le contexte américain, Lortie (1975, éd. 2002) avait déjà mis en évidence que le choix de l'enseignement se construisait, durant la scolarité des individus, au travers d'enseignants modèles. Ce "phénomène de continuation ", qui est une forme de socialisation primaire spécifique (Charles, 2012), est récurrent dans ces trajectoires directes. Cependant, ceux qui choisissent l'enseignement primaire trouvent leur inspiration dans leur propre scolarité au niveau primaire, avec des années au secondaire peu notable, voire marquées par des difficultés. A contrario, ceux qui choisissent l'enseignement secondaire ont été marqués par des enseignants qui ont cru en eux durant cette période d'adolescence. Ce phénomène se traduit également par une interaction qui sera maintenue diachroniquement avec l'enseignant porteur de valeurs de référence. Pour certains, il jouera même un rôle au moment de s'essayer au métier, en allant notamment intervenir dans sa classe en tant que stagiaire avant de choisir la formation à l'enseignement.

\section{L'enseignement par " socialisation anticipatrice »}

Les mécanismes d'identification au groupe de référence qui interviennent alors que les enseignants sont parfois très jeunes ne suffisent pas pour expliquer une passion qui perdure, notamment pour les personnes qui déclarent vivre l'enseignement primaire comme une vocation depuis leur enfance. Cette vocation s'accompagne également d'activités qui participent à la socialisation anticipatrice (Charles, 2012) dès l'adolescence. Celles qui enseignent au primaire ont fait du babysitting, des stages, donné des leçons d'appui ou ont été impliquées

\footnotetext{
4. Les proportions d'hommes et de femmes dans chaque formation se reflètent dans une certaine mesure par ces chiffres. Actuellement, neuf enseignantes sur dix sont des femmes au niveau primaire, tandis que le niveau secondaire est plus proche de la parité.

5. Après une scolarité obligatoire dans la voie dite supérieure à l'époque, elle a débuté ses études de niveau secondaire 2 dans la voie générale qui ne donne pas accès directement à l'université. Elle a donc dû passer deux ans de plus - cinq ans de secondaire 2 , afin d'obtenir sa maturité fédérale.
} 
dans des camps de vacances ou dans leur club sportif et «se sentent à l'aise avec les enfants ». Pour celles qui enseignent dans le secondaire, l'orientation vers l'enseignement passe également par une hiérarchisation des préférences parmi les différents "jobs» d'étudiants exercés entre la fin de l'école obligatoire et l'obtention de leur diplôme universitaire. Les quelques heures de remplacement ou encore les cours d'appui ont toujours été préférés aux autres activités précaires exercées.

\section{L'enseignement comme " rapport au savoir"}

Dans le contexte français, l'analyse des déterminants d'accès à l'enseignement secondaire par Deauvieau (2005) met en évidence la bonne scolarité des futurs enseignants du secondaire, qui les amène alors à entretenir un rapport « intellectuel » à une discipline. C'est notamment ce "rapport au savoir " qui les oriente vers des disciplines académiques, au détriment d'une formation pratique. Dans nos cas, nous constatons que cette lecture chronologique du « rapport au savoir» (Deauvieau, 2005) passe d'abord par l'origine sociale des différentes trajectoires linéaires. En effet, une particularité des récits des enseignantes du primaire par rapport aux enseignants du secondaire réside dans la formation et le métier de leurs parents, plus particulièrement de leur mère, comme constaté par Denzler et Wolter (2009). Cette différence a également des conséquences pratiques. Là où certains parents hautement formés interviennent très peu, sinon en tant que soutien dans les orientations, parce que leur enfant poursuit la voie de longues études, d'autres parents conseillent une formation orientée vers un métier concret. Mais cette reproduction du " rapport au savoir » s'écarte de la description qui en est faite par Deauvieau, dans la mesure où ceux qui atteignent l'Université le font par défaut, sans y exceller. Leur diplôme universitaire est décrit comme étant une limite dans un rapport plutôt scolaire du savoir, c'est-à-dire la capacité à restituer le savoir dispensé. Pour celles qui s'orientent vers le primaire, les difficultés face au savoir s'éprouvent durant leur passage au secondaire 2, ce qui participe à la dissuasion d'une prolongation des études par un cursus universitaire.

\section{LES TRAJECTOIRES INDIRECTES VERS L'ENSEIGNEMENT}

Les enseignants ayant un parcours indirect vers l'enseignement se caractérisent par les orientations choisies lors de deux transitions clés durant leur formation : soit la suite donnée à leur formation de niveau secondaire 2 - choix qui seront abandonnés au profit d'une formation pour enseigner au primaire, soit l'incertitude professionnelle à la fin de leur formation universitaire, qui trouvera sa résolution dans la formation pour l'enseignement secondaire. Cette incertitude professionnelle qui pèse sur leurs trajectoires, durant un certain 
temps, s'observe objectivement: d'une part dans la composition de cette catégorie - on y retrouve notamment deux hommes qui ont suivi la formation pour enseigner au primaire, d'autre part avec un âge médian plus élevé. Les six enseignants de degré primaire terminaient leur formation en ayant 26 ans d'âge médian, tandis que parmi les quatre enseignants au bénéfice d'une formation pour l'enseignement secondaire, la plus jeune avait 29 ans et la plus âgée 41 ans.

Cependant, leurs trajectoires comportent des similitudes avec ceux qui s'orientent directement vers le métier. Dans la plupart des cas, les scolarités sont marquées par une figure enseignante qui leur sert de modèle d'orientation. Leur " rapport au savoir» rythme leur parcours de formation. Finalement, ils sont caractérisés par un premier projet professionnel contrarié par les prérequis d'accès à celui-ci.

\section{Des modèles sources de dissonance}

Mais les différences se vivent notamment dans les effets de la socialisation primaire spécifique. En effet, pour quelqu'un comme Philippe ${ }^{6}$, qui est issu, selon ses termes, "d'une dynastie d'enseignants ", le métier de ses deux parents a été vécu avec considération, mais également des réserves. Il a passé sa scolarité à vivre les "coulisses de l'enseignement " non seulement à la maison mais également dans l'ombre de son nom de famille, étant donné qu'il a passé sa scolarité secondaire dans le collège où exerçait son père - donc avec sa réputation qui le poursuivait dans la cour de récréation ou dans le bus, et en choisissant la même option de formation que lui. Cela ne l'a pourtant pas empêché d'envisager le métier durant toute sa scolarité, et d'exercer de longues heures en tant que remplaçant, avant de s'essayer à autre chose durant et après ses études universitaires. L'enseignement reviendra comme une évidence à la suite de différentes désillusions vécues sur le marché de l'emploi.

Pour d'autres, la scolarité n'a pas toujours été valorisée et les enseignants modèles rencontrés ne les ont pas attirés vers ce métier en particulier, mais les ont poussés à s'ouvrir à d'autres métiers, d'autres passions comme c'est le cas pour Rita, qui trouve un soutien auprès d'un enseignant d'art visuel qui va l'inciter à élaborer un projet professionnel autour de la création artistique. Parallèlement, la relation avec sa grand-mère l'incite à s'intéresser de plus en plus à cette création dans l'univers de la mode. Mais la formation universitaire dans le domaine, et les autres voies d'accès notamment par le travail salarié ne la convainquent pas. Elle cherche à reprendre les études et se rend compte qu'elle a pris plaisir aux activités ayant contribué à sa socialisation anticipatrice de l'enseignement. Ainsi, ces modèles véhiculant un certain nombre de valeurs les ont plutôt orientés vers une

6. Les prénoms utilisés pour faire référence aux participants à la recherche sont fictifs. 
forme d'incertitude ou vers un premier projet professionnel, jusqu'à ce qu'ils s'orientent vers l'enseignement, suite aux désillusions vécues ou à défaut de projet convaincant.

\section{« Un rapport au savoir " contrarié}

La présence d'un modèle parental ou d'un enseignant influe notamment sur le choix d'un parcours et d'un projet professionnel nécessitant de longues études. Pourtant, des difficultés contextuelles éprouvent ces parcours scolaires de plusieurs manières, comme le changement du contexte de vie par une décision relevant du cercle ${ }^{7}$ familial - déménagement, migration ou changement dans le contexte scolaire. Une première distinction de ces parcours s'observe à la fin du secondaire 2. Il y a d'une part ceux qui arrivent à faire la transition de la forme scolaire à la forme académique et, d'autre part, ceux qui s'y essaient ou font une tentative d'insertion sur le marché du travail, mais sans succès dans ces deux derniers cas.

Les premiers, ceux qui finiront notamment par choisir l'enseignement au niveau secondaire, s'adaptent parfaitement à l'univers académique. Ils vivent de manière optimale l'autonomie de travail, la flexibilité dans le fonctionnement et la "relativité » des savoirs. En effet, la gestion du temps académique leur permet, entre autres, de s'essayer au métier d'enseignant remplaçant, tout en s'émancipant de leur cercle familial - en s'installant en couple. Cette aisance transformera ce "rapport au savoir » en un projet professionnel orienté vers la recherche pour deux des quatre cas analysés. Mais le manque d'opportunité ainsi que la désillusion par rapport aux autres débouchés, à la suite d'un long parcours universitaire, les mèneront vers la formation pour l'enseignement secondaire.

Ceux qui optent pour l'enseignement primaire se singularisent par leur premier projet professionnel, qui se définit au moment de leur scolarité post obligatoire, au secondaire 2. Pour deux d'entre eux (dont Rita précédemment citée), leur première orientation professionnelle ne nécessitait pas un passage par l'université. Mais ils vont essuyer une désillusion sur le marché de l'emploi, car ils n'y trouvent pas le débouché recherché. L'enseignement comme alternative face à l'incertitude professionnelle est une réévaluation de leur parcours, si l'on considère notamment les éléments (personnes, activités) ayant participé à leurs socialisations spécifique et anticipatrice. Il y a également ceux qui choisissent d'entrer à l'université, notamment pour cultiver une certaine facilité d'apprentissage éprouvée jusqu'alors. L'enseignement au niveau secondaire est l'un de leur projets professionnels potentiels. Pourtant, ils n'arriveront pas à s'adapter à la forme académique et vont essuyer un échec et une désillusion dans ce nouveau cercle. L'enseignement au niveau primaire sera une adaptation de leurs ambitions de formation et de projet professionnel.

7. Sur la notion de cercle, voir Grossetti et Bes, 2003. 


\section{L'ENSEIGNEMENT COMME UN SECOND MÉTIER}

Pour ceux qui choisissent l'enseignement en tant que second métier, une telle réorientation est d'abord à mettre en relation avec le(s) emploi(s) qui précèdent l'enseignement, ou plutôt la première formation qu'ils ont suivie. C'est en effet celle-ci qui a rendu possible l'exercice d'une ou des activités rémunérées sur une période plus ou moins longue, avant la formation d'enseignant. Cela explique notamment l'hétérogénéité de trajectoires qui composent cette catégorie, en termes de lien entre le cycle de la vie biologique et le cycle de la vie sociale. D'une part, ces premières formations majoritairement universitaires (12 sur les 15 individus) sont très diverses, bien que ce soient celles-ci qui rendent possible la formation à l'enseignement secondaire par la suite. D'autre part, la durée des emplois ayant suivi cette première formation est très variable. Ainsi, la personne la plus âgée a travaillé dans l'industrie privée comme ingénieur pendant 28 ans, alors que la plus jeune a 30 ans et a exercé comme comédienne indépendante pendant deux ans, avant d'orienter son projet professionnel vers l'enseignement.

Cependant, la subdivision qui fait le plus sens, d'un point de vue analytique, est ailleurs. En l'occurrence, la typologie qui ressort de notre analyse différencie les carrières selon deux axes imbriqués. Le premier axe préfigure la relation de continuité - rupture entre la ou les activités professionnelles exercées avant de s'orienter vers l'enseignement et l'enseignement. Elle discerne trois positions : la conciliation professionnelle, l'adaptation professionnelle et la rupture professionnelle. Le second axe interroge la place subjective du nouveau projet professionnel - l'enseignement - en le situant dans l'ensemble des activités qui font sens au moment du choix de l'enseignement. L'enseignement peut être une activité de complément, d'aménagement ou de changement.

\section{Lien entre l'enseignement et les activités précédentes}

Les approches sociologiques les plus répandues pour examiner le changement de métier considèrent surtout les ruptures professionnelles, qui sont à mettre en lien avec le chômage systémique qui touche l'économie globalisée. En exemple, l'approche interactionniste des reconversions professionnelles de Denave (2006) propose cinq phases ${ }^{8}$ pour caractériser les mécanismes à l'œuvre

8. Les individus en reconversion professionnelle vivent d'abord un décalage qui s'exprime de deux manières. Soit ce décalage a toujours existé, soit il s'est constitué petit à petit durant l'exercice de l'emploi qui a précédé la réorientation professionnelle. La deuxième phase est marquée par des événements déclencheurs qui peuvent tout autant provenir de la vie professionnelle que de la vie privée de l'individu. Le désengagement de l'emploi constitue la troisième phase de ce processus de reconversion professionnelle. Son déroulement est fonction des ressources socioéconomique à disposition. Une fois la personne désengagée totalement de l'activité précédente, alors elle s'oriente vers des aspirations plus en résonance avec sa situation, ce qui marque la quatrième phase. Elle se déroule dans l'informel. Finalement, la dernière phase vient en opposition au désengagement. Elle dépend également des ressources à disposition de la personne, qui vont lui permettre de suivre la nouvelle formation professionnelle vers un nouveau métier. 
dans le processus de désengagement du premier métier et d'engagement dans le second. Ces phases sont utiles notamment dans une première lecture des trajectoires qui se caractérisent par l'exercice d'un premier métier. Mais le recours à la littérature sur les transitions professionnelles, à l'instar des situations analysées par D’Ascoli et Berger (2012), est également indispensable pour mettre en lumière la particularité du choix de l'enseignement comme nouvelle activité.

La conciliation professionnelle capture l'ensemble des trajectoires où la transition professionnelle n'est possible que par le lien explicite qui existe entre l'enseignement et les activités précédentes qui se maintiennent en partie. Elle caractérise les parcours d'artistes indépendants, comédiens et musiciens professionnels qui stabilisent leur situation financière à l'aide de l'enseignement, mais poursuivent leur première activité. L'adaptation professionnelle correspond à la valorisation de certaines compétences et tâches des activités précédentes, comme la gestion d'une équipe d'apprentis dans une usine, qui permet d'appréhender différemment la gestion de classe. Enfin, la rupture professionnelle caractérise les reconversions radicales où aucun lien ne subsiste entre l'enseignement et les tâches et activités précédentes.

\section{L'enseignement comme une activité parmi d'autres}

Dans l'analyse du choix de l'enseignement comme second métier (D’Ascoli \& Berger, 2012), l'analyse des motivations éclaire en partie les spécificités de l'enseignement, notamment les avantages qu'une telle activité comporte selon «l'état de carrière » considéré. Dès lors, nous envisageons l'enseignement comme une activité de complément dans les cas où elle a une utilité personnelle, plus précisément dans la gestion d'autres activités professionnelles moins rémunératrices et moins stables. C'est notamment le cas pour un musicien instrumentiste, qui a longtemps gagné sa vie en jouant dans divers groupes professionnels et qui cherche à stabiliser son expertise en tant qu'enseignant de musique tout en diminuant ses tournées. L'enseignement sera vécu comme une activité d'aménagement pour les personnes qui cherchent à concilier l'ensemble des cercles sociaux dans lesquelles elles sont impliquées. De fait, la flexibilité de l'enseignement offre plus de compatibilité avec un engagement plus ou moins intense dans la parentalité. Mais il existe également des personnes qui vivent l'enseignement comme une activité de changement et pour lesquelles le métier correspond à ce que Négroni (2005) définit comme une "conversion de soi ».

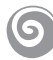

Comment s'oriente-t-on vers l'enseignement? Au travers de cet article, nous avons souligné les caractéristiques des trois parcours types qui mènent vers la formation à l'enseignement dans le canton de Vaud, en Suisse. Les premiers 
parcours dits "directs " correspondent à ceux qui font l'objet de la majeure partie de la littérature sur la profession enseignante. Ces derniers se sont formés expressément pour devenir enseignants. Dans le contexte suisse romand, le choix du métier à la suite de ce type de parcours comprend trois dimensions : l'identification à un sous-groupe de référence (enseignants du primaire ou enseignants du secondaire) biographiquement situé ; une socialisation à l'activité de transmission et de soutien à un public particulier (les enfants d'un certain âge) ; et un rapport social au savoir à transmettre.

Comparativement, la singularité des individus au parcours indirect est surtout d'ordre temporel. En effet, l'incertitude qui pèse sur leur trajectoire est à mettre en lien avec un premier projet professionnel, plus ou moins flou, dont la source n'est autre que le rapport au savoir construit tout au long de leur scolarité. Mais c'est bien parce qu'ils reconnaissent rétrospectivement certains enseignants et certaines activités anticipatrices du métier dans leur parcours de vie et de formation qu'ils s'orientent finalement vers l'enseignement.

La dernière catégorie regroupe des parcours d'individus qui se sont formés à d'autres métiers qu'ils ont exercés. Pour ces derniers, deux dimensions éclairent leur choix : le lien entre l'enseignement et leur passé professionnel ; et la place ou le rôle de cette nouvelle orientation dans leur présent. L'enseignement se vit alors en continuité ou en rupture avec leur précédente activité, avec l'adaptation de certaines compétences comme position intermédiaire (une forme de socialisation anticipatrice). Parallèlement, le métier aura une certaine utilité dans l'agencement du quotidien : par la stabilité professionnelle qu'il apporte à d'autres projets professionnels plus instables; dans la gestion des activités qui font sens pour l'individu, ou comme source de conversion de soi.

Ces trois parcours types - « direct », « indirect » et « second métier »correspondent aux trois " états de carrière » qui caractérisent les individus avant leur formation à l'enseignement. Pour conclure, nous pourrions nous demander quel est l'impact de chaque type de trajectoire sur le processus d'insertion professionnelle énoncé en introduction. Nos analyses tendent à montrer qu'après la formation, les parcours atypiques accèdent différemment à l'emploi recherché. Contrairement aux enseignants avec un parcours direct qui raisonnent étape par étape, ceux qui ont un parcours atypique (surtout la catégorie " second métier») auraient tendance à privilégier l'emploi. De par leur état de carrière et les responsabilités qui leur incombent, ils préfèrent effectuer leur formation en occupant un emploi sous forme de stage à responsabilité ${ }^{9}$ dans un établissement. Le réseau qu'ils y créent leur facilite l'accès aux postes qui correspondent à ce qu'ils recherchent à la fin de la formation. Dans le cas où ils sont engagés dans le même établissement, les enjeux de l'insertion subjective (intégration et collaboration

9. La formation en alternance accepte deux types de stages : le stage dans une classe d'un praticien formateur et le stage à responsabilité ou en autonomie dans sa propre classe. Ce dernier garantit un meilleur salaire et un accès privilégié à l'informel de l'établissement. 
dans l'équipe pédagogique, maîtrise des tâches périphériques à l'activité centrale "l'enseignement», gestion des difficultés au quotidien) s'en trouvent adoucis. Ainsi le processus d'insertion n'est pas linéaire. Cependant, c'est également l'existence d'un autre projet professionnel contrarié ou d'une autre activité avant l'enseignement qui serait susceptible de moduler l'engagement / désengagement dans le métier.

\section{BiBLIOGRAPHIE}

CHARLES F. (2012) : "Les différents types de socialisation à l'œuvre dans le recrutement des professeurs du secondaire en Angleterre et en France ", in P. Guibert et P. Périer (Éd.), La socialisation professionnelle des enseignants du secondaire : parcours, expériences, épreuves (p. 17-40). Rennes: Presses universitaires de Rennes.

D’ASCOLI Y., BERGER J.-L. (2012) : "Les déterminants du choix de carrière des enseignants de la formation professionnelle et leur relation aux caractéristiques sociodémographiques ", Nouveaux c@hiers de la recherche en éducation, 15(2), 1.

DEAUVIEAU J. (2005) : Devenir enseignant du secondaire : les logiques d'accès au métier, Revue française de pédagogie, 31-41.

DENAVE S (2006) : "Les conditions individuelles et collectives des ruptures professionnelles ", Cahiers internationaux de sociologie, (120), 85-110.

DENZLER S., WOLTER S. C. (2009) : "Sorting into teacher education: how the institutional setting matters ", Cambridge Journal of Education, 39(4), 423-441.

GIRINSHUTI C. (2014) : L'insertion professionnelle des enseignants : comment devient-on enseignant?, canevas de thèse accepté le 8 mai 2014, Université de Genève.

GROSSETTI M., BES M.-P. (2003) : « Dynamiques des réseaux et des cercles. Encastrements et découplages ", Revue d'économie industrielle, 103(1), 43-58.

HUGHES E. C. (1996) : Le regard sociologique : essais choisis, Paris : Éd. de l'École des hautes études en sciences sociales.

LORTIE D. (1975, éd. 2002) : Schoolteacher: A sociological study. Chicago : University of Chicago Press.

NÉGRONI C. (2005) : «La reconversion professionnelle volontaire : une expérience de conversion de soi ", Revue Carriérologie, 10(2). 